\title{
Electroweak and QCD corrections to $Z$-boson production with one $b$ jet in a massive five-flavor scheme
}

\author{
D. Figueroa, ${ }^{1, *}$ S. Honeywell, ${ }^{1, \dagger}$ S. Quackenbush,${ }^{1, \ddagger}$ L. Reina, ${ }^{1,}$ C. Reuschle, ${ }^{1,2, \|}$ and D. Wackeroth ${ }^{3, \Uparrow}$ \\ ${ }^{1}$ Physics Department, Florida State University, Tallahassee, Florida 32306-4350, USA \\ ${ }^{2}$ Department of Astronomy and Theoretical Physics, Lund University, 22362 Lund, Sweden \\ ${ }^{3}$ Department of Physics, SUNY at Buffalo, Buffalo, New York 14260-1500, USA
}

(Received 26 June 2018; published 5 November 2018)

\begin{abstract}
We compute the $O\left(\alpha_{s} \alpha^{2}\right)$ and $O\left(\alpha_{s}^{2} \alpha\right)$ contributions to the production cross section of a $Z$ boson with one $b$ jet at the Large Hadron Collider (LHC) and study their phenomenological relevance for LHC physics. The accurate prediction of hadronic $Z+b$-jet production is needed to control a background that greatly affects both the measurement of Higgs-boson properties and the searches of new physics at the LHC. At the same time it could enable the first precise measurement of the $b$-quark parton distribution function. In this context $b$-quark mass effects become relevant and need to be studied with care, both at the level of the hard process and at the level of the initial- and final-state parton evolution. It is the aim of this paper to explore some of these issues in the framework of a massive five-flavor scheme and to assess the need for the inclusion of both electroweak corrections, in addition to QCD corrections, and $b$-quark mass effects in the prediction of total and differential cross sections for hadronic $Z+b$-jet production.
\end{abstract}

DOI: 10.1103/PhysRevD.98.093002

\section{INTRODUCTION}

The production of a $Z$ boson with one or more $b$ jets plays a very important role in the physics program of the Large Hadron Collider (LHC) both for direct searches of physics beyond the Standard Model (SM) and for precision measurements of SM processes that could reveal deviations induced by new physics beyond the direct reach of the LHC. Dedicated experimental studies from the LHC, which upgraded previous Tevatron results [1,2], have been published during Run I [3-9], and we look forward to results obtained at higher center-of-mass (c.m.) energy and with much higher statistics during Run II.

$Z+b$ jet(s) is an important reducible and irreducible background to several SM and beyond the SM (BSM) processes involving $Z$ bosons and jets. Indeed, signals of physics beyond the SM will likely emerge from signatures containing heavy SM particles, such as $Z$ and $W$ bosons, together with $t$ and $b$ quarks. On the other hand, in the case,

\footnotetext{
daf14f@my.fsu.edu †sjh07@hep.fsu.edu

*squackenbush@hep.fsu.edu

\$reina@hep.fsu.edu

"creuschle@hep.fsu.edu

christian.reuschle@thep.lu.se

dow@ubpheno.physics.buffalo.edu
}

Published by the American Physical Society under the terms of the Creative Commons Attribution 4.0 International license. Further distribution of this work must maintain attribution to the author(s) and the published article's title, journal citation, and DOI. Funded by SCOAP ${ }^{3}$. for instance, of SM Higgs production, $Z+b$ jet(s) is the dominant background to the $Z H$ associated production mode, with the Higgs decaying into a $b \bar{b}$ pair. The theoretical accuracy of the prediction of $Z+b$ jets will therefore affect the precision on measurements of Higgsboson couplings reached at the LHC.

Besides searches for new physics, the interest in a precision measurement of $Z+b$-jet production is also motivated by the possibility of obtaining the first direct measurement of the $b$-quark parton distribution function (PDF). Indeed, if one assumes a nonzero $b$-quark PDF, i.e., if one works in a five-flavor scheme (5FS), the production of a $Z$ boson with one $b$ jet proceeds dominantly via $b g \rightarrow Z b$. If this is very natural at energy scales much larger than $m_{b}$, it becomes less justified at lower energies where working in a four-flavor scheme (4FS) may also be appropriate, and the channels $q \bar{q} \rightarrow Z b \bar{b}$ and $g g \rightarrow Z b \bar{b}$ are the main channels for $Z$ production with both one and two $b$ jets. In the past few years a lot of theoretical activity has gone into clarifying the interplay between the two approaches [10-18] (for a review see also Ref. [19]), and times are now mature to develop a dedicated experimental program aimed at the measurement of the $b$-quark parton density via processes such as $Z+b$ jets, or $\gamma+b$ jets, which provides analogous yet complementary information.

As already proven by the incredibly successful physics program of Run I of the LHC, both the intricacy of new physics searches and the challenge of SM precision measurements require the improvement of the accuracy of theoretical predictions to the percent level. If the knowledge of the first order(s) of QCD corrections was 
mandatory for Run I, electroweak (EW) corrections will also become important at the energies of Run II. At the same time, a more accurate assessment of the theoretical uncertainties intrinsic to sophisticated Monte Carlo tools used to match higher-order $\mathrm{QCD} / \mathrm{EW}$ calculations to parton-shower generators is clearly needed. Among others, the question of properly including heavy-quark mass effects ( $b$-quark mass effects in our case), both at the level of the hard-scattering matrix element and at the level of the PDF, should be carefully considered, in particular for $b$-quark initiated processes. Given its relevance for the physics of Run II, $Z+b$-jet production offers a particularly interesting prototype case to be considered in this context.

With this in mind, we consider in this paper the production of a $Z$ boson with one $b$ jet as generated at leading order (LO) via the tree-level $O\left(\alpha_{s} \alpha\right)$ process $b g \rightarrow Z b$. The first order of QCD corrections, i.e., the $O\left(\alpha_{s}\right)$ or next-to-leading order (NLO) QCD corrections to this process, have been calculated for the first time in Ref. [20] and implemented in MCFM [21], assuming a massless $b$ quark. To improve the precision of the NLO QCD theoretical predictions one could add either the second order [i.e., the next-to-next-to-leading order (NNLO)] QCD corrections or the first order (i.e., the NLO) EW corrections. Adding NNLO QCD corrections should certainly stabilize the cross section by reducing the dependence on the renormalization and factorization scales, while adding NLO EW corrections could add a few percent to total rates and have a visible impact on high-energy tails of distributions. NNLO QCD corrections to $Z+$ jets have been presented in Refs. [22,23] and, with due care, they could be used in the future to extract the NNLO QCD prediction for $Z+b$-jet for the case of a massless $b$ quark. The $O\left(\alpha_{s}\right)$ corrections to $q \bar{q}, g g \rightarrow Z b \bar{b}$ for a massive $b$ quark have also been calculated [24,25]. They represent an important component of the NNLO QCD corrections for massive $b$ quarks and indicate a sensible reduction of the overall scale dependence for $Z+b$ jet production. Obtaining NNLO QCD corrections for a massive $b$ quark is a more challenging endeavor and should be considered only after the inclusion of massive initial-state partons, some issues of which we discuss in this paper. NLO EW corrections to hadronic $Z+j$ production have been presented in Refs. [26-29], and combined NLO QCD + EW corrections in Ref. [30]. The first order of EW corrections to $Z+b$-jet production via $b g \rightarrow Z b$, i.e., the $O\left(\alpha_{s} \alpha^{2}\right)$ term in the perturbative expansion of the cross section for $Z+b$-jet production, is the main subject of this paper, where we also study the effect of considering an initial-state massive $b$ quark and discuss the interplay with the definition of the corresponding $b$-quark PDF. This can be considered as the first building block of a more general program that will have to connect both 4FS and 5FS calculations, including both QCD and EW corrections.
Since the EW corrections to $b g \rightarrow Z b$ represent a well defined set of corrections, in a well defined flavor scheme, this calculation allows one to estimate in a consistent way the impact of EW corrections on $Z+b$-jet production through the process that also most affects the measurement of the $b$-quark PDF.

Furthermore, working with the idea of implementing a 5FS calculation in a parton-shower Monte Carlo event generator, we treat the $b$ quark as massive also in the initial state, since this is necessary in order to properly implement the backward evolution of final-state massive $b$ quarks. Indeed, if it is customary to treat initial-state $b$ quarks as massless (traditional 5FS), this is nevertheless just a simplification of the calculation, not a requirement [31-33]. More to the point, this approximation does not lend itself well to the implementation of methods, such as phase-space slicing methods or subtraction methods, which evaluate higher-order real radiative corrections with the help of auxiliary terms that algorithmically approximate the realemission contributions in the soft/collinear regions starting from the corresponding Born processes. For example, a real emission subtraction term to the real-emission process $g g \rightarrow Z b \bar{b}$, with massive $b$ quarks, cannot be generated in a kinematically consistent way from the corresponding Born process $b g \rightarrow Z b$, with massless $b$ quarks, by convoluting with the splitting function for $g \rightarrow b \bar{b}$. Since it is, for example, at the core of the implementation of 5FS processes in Monte Carlo event generators that match NLO cross sections to parton showers, this issue has recently attracted some attention and studies aimed at introducing what has been dubbed as massive 5FS (m5FS ${ }^{1}$ ) have appeared [34]. Hence, in view of future developments in Monte Carlo event generators, we consistently develop the calculation of both the first order of QCD and EW corrections with a massive $b$ quark. This incidentally also implies that we extend the existing NLO QCD calculation [20] to the case of a massive $b$ quark.

In this paper, both QCD and EW virtual corrections have been obtained through the NLOX one-loop provider [35], as well as by independent in-house codes. The corresponding real corrections have been computed, in both the massless and massive cases, via independent codes using phasespace slicing, as well as an implementation of the dipole subtraction method based on the formalism of Ref. [36], extended to QED radiation off massive quarks (in both initial and final states). More details will be presented in Sec. II. Having obtained both the $O\left(\alpha_{s}\right)$ and the $O(\alpha)$ corrections to $b g \rightarrow Z b$ including full $b$-quark mass effects, we can assess the following: (1) the impact of mass effects on the fixed-order total cross section and distributions by

\footnotetext{
${ }^{1}$ So far we have used 5FS to denote a generic scheme with 5 active flavors. In the following we will use 5FS to denote the case in which the $b$ quark is considered massless, and m5FS to denote the case in which the $b$ quark is considered massive.
} 
comparing NLO QCD cross sections with massless and massive $b$ quarks, and (2) the relative impact of QCD and EW corrections on fixed-order total cross sections and distributions by comparing the $O\left(\alpha_{s}^{2} \alpha\right)$ and $O\left(\alpha_{s} \alpha^{2}\right)$ cross sections with massive $b$ quarks. Indeed, independent of the necessity of introducing massive initial-state partons for automated implementations of NLO QCD and EW corrections, it is also clear that mass effects and EW corrections can be of the same order, although they typically affect physical observables in different kinematical regions. Even if small, they both need to be accounted for when one aims for percent-level precision predictions over a broad kinematical range.

In the remainder of this paper we systematically review in Sec. II the relevant technical details of the calculation and present results in Sec. III where we also assess the impact of such corrections and discuss the need for future improvements. Conclusions and suggestions for future developments are presented in Sec. IV.

\section{DETAILS OF THE CALCULATION}

We write the hadronic cross section, $\sigma$, for $Z+b$-jet production at the LHC as follows:

$$
\sigma=\sum_{i, j} \alpha_{s}^{i} \alpha^{j} \sigma^{(i, j)}
$$

where $i$ and $j$, with $i+j \geq 2$ and $j \geq 1$, refer to the coupling order of the partonic cross section, and $\sigma^{(i, j)}$ denotes the term in the perturbative expansion of the cross section that is proportional to $\alpha_{s}^{i} \alpha^{j}$ [where $\alpha_{s}=g_{s}^{2} /(4 \pi)$ and $\alpha=e^{2} /(4 \pi), g_{s}$ and $e$ being the QCD and QED coupling constants, respectively]. In a scheme with five active flavors (such as the 5FS or m5FS), the set of "lowestorder" contributions with $i+j=2$ consists of all sets of tree-level diagrams that satisfy such a relation; i.e., $\sigma^{(1,1)}$ corresponds to the tree-level contributions to $b g \rightarrow Z b$ and $\sigma^{(0,2)}$ to the tree-level contributions to $b \gamma \rightarrow Z b .^{2}$ It is clear that the calculation of the cross section also includes the corresponding $\bar{b} g$-initiated and $\bar{b} \gamma$-initiated processes, even if it is not explicitly repeated throughout the paper. Both processes consist of $s$ - and $t$-channel contributions, as illustrated in Fig. 1 for the case of $b g \rightarrow Z b$ (the corresponding diagrams for $b \gamma \rightarrow Z b$ are obtained by replacing a gluon with a photon in both diagrams).

The set of "next-to-lowest-order" contributions consists of the $O\left(\alpha_{s}\right)$ and $O(\alpha)$ corrections to the set of "lowest-order" contributions, as depicted in Fig. 2. The $O\left(\alpha_{s}^{2} \alpha\right)$ term, $\sigma^{(2,1)}$, corresponds to the $O\left(\alpha_{s}\right)$ corrections to

\footnotetext{
${ }^{2}$ Note that the set of all possible lowest-order contributions is made of contributions of different coupling-power combinations and that those also arise from different sets of initial-state particles. The same holds for the set of all possible next-tolowest-order contributions.
}

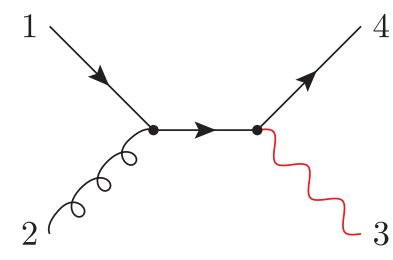

(a)

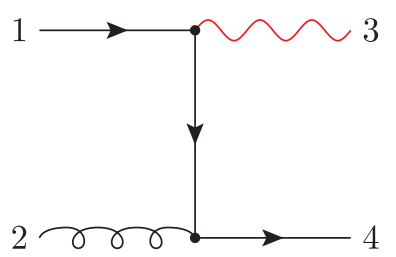

(b)
FIG. 1. Tree-level ( $s$ - and $t$-channel) Feynman diagrams for $b(1) g(2) \rightarrow Z(3) b(4)$.

$\sigma^{(1,1)}$. It was first computed in Ref. [20] for massless $b$ quarks, and the corresponding calculation is implemented in MCFM [21]. We have reproduced it independently in this paper, where we also extend it to the case of a massive $b$ quark in order to consistently compare the impact of NLO QCD and EW corrections, and in order to assess nonzero $b$-quark mass effects by comparing massive and massless NLO QCD results. The $O\left(\alpha_{s} \alpha^{2}\right)$ term, $\sigma^{(1,2)}$, is also presented for the first time in this paper and is indeed the main focus of our study. Such a term originates from both the $O(\alpha)$ corrections to $\sigma^{(1,1)}$ $(b g \rightarrow Z b)$ and the $O\left(\alpha_{s}\right)$ corrections to $\sigma^{(0,2)}$ $(b \gamma \rightarrow Z b)$, as depicted in Fig. 2. It is entirely dominated by the $O(\alpha)$ corrections to the $b g \rightarrow Z b$ tree-level process, since the cross section for $b \gamma \rightarrow Z b$ is much smaller due to the smallness of the photon parton density in the initial-state protons. ${ }^{3}$ Hence in our study we will only consider the $O(\alpha)$ corrections to $b g \rightarrow Z b$. Also the $O\left(\alpha^{3}\right)$ term, $\sigma^{(0,3)}$, is entirely negligible and will not be considered here. We will thus from here on simply speak of $\sigma^{(1,1)}$ as the LO contribution, and define

$$
\alpha_{s} \alpha \sigma^{(1,1)}+\alpha^{2} \sigma^{(0,2)} \approx \alpha_{s} \alpha \sigma^{(1,1)} \equiv \sigma_{\mathrm{LO}},
$$

and of $\sigma^{(2,1)}$ and $\sigma^{(1,2)}$ as the $O\left(\alpha_{s}\right)$ and $O(\alpha)$ corrections, or simply NLO QCD and EW corrections, respectively, and define the corresponding NLO QCD and NLO EW cross sections as

$$
\begin{gathered}
\sigma_{\mathrm{NLO}}^{\mathrm{QCD}} \equiv \sigma_{\mathrm{LO}}+\alpha_{s}^{2} \alpha \sigma^{(2,1)}, \\
\sigma_{\mathrm{NLO}}^{\mathrm{EW}} \equiv \sigma_{\mathrm{LO}}+\alpha_{s} \alpha^{2} \sigma^{(1,2)} .
\end{gathered}
$$

In order to implement both the NLO EW and NLO QCD cross sections in the m5FS, the full $b$-quark mass dependence has been retained both in the hard-scattering matrix

\footnotetext{
${ }^{3}$ Using the setup described in Sec. III, one finds that $\sigma_{\mathrm{LO}}=\alpha_{s} \alpha \sigma^{(1,1)} \simeq 376 \mathrm{pb}$, while the cross section for the lowestorder photon-induced process is 3 orders of magnitude smaller, $\alpha^{2} \sigma^{(0,2)} \simeq 0.1 \mathrm{pb}$, and 1 order of magnitude smaller than $\alpha_{s} \alpha^{2} \sigma^{(1,2)} \simeq 5 \mathrm{pb}$.
} 


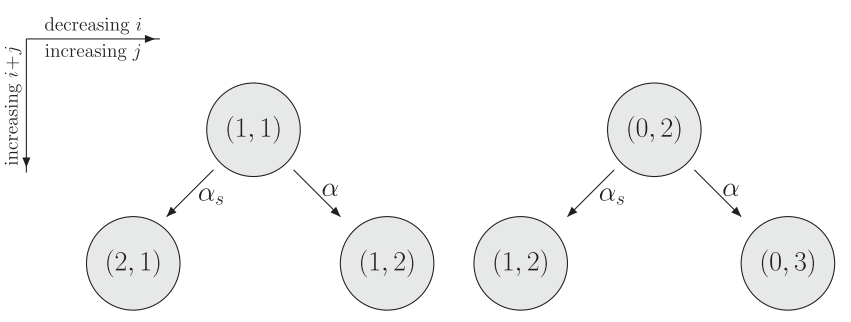

FIG. 2. Coupling-power flow chart for $Z+b$-jet production at the level of the partonic cross section. The notation $(i, j)$ corresponds to the coupling-power combination $\alpha_{s}^{i} \alpha^{j}$. From left to right we have increasing $i$ /decreasing $j$ in steps of 1 . From top to bottom the total order $i+j$ increases in steps of 1 . The upper row depicts all possible coupling-power combinations for the "lowest-order" contributions, with $i+j=2$, while the lower row depicts all possible couplingpower combinations for the higher-order corrections of one order higher, with $i+j=3$. Note that the $(1,2)$ contribution is depicted twice, as it originates from two different lowest-order contributions with different sets of initial-state particles.

elements and in the phase-space integration. We therefore have defined the initial-state parton-level kinematics of $b\left(p_{b}\right)+g\left(p_{g}\right) \rightarrow Z b$ as follows [37]:

$$
\begin{aligned}
p_{b}^{\mu}= & \frac{\sqrt{s}}{2}\left(x_{1}+\frac{m_{b}^{2}}{x_{1} s}, 0,0, x_{1}-\frac{m_{b}^{2}}{x_{1} s}\right) \\
& \text { with } \quad \frac{m_{b}}{\sqrt{s}} \leq x_{1} \leq \frac{1}{2}\left(1+\sqrt{1-\frac{4 m_{b}^{2}}{s}}\right), \\
p_{g}^{\mu}= & \frac{\sqrt{s}}{2}\left(x_{2}, 0,0,-x_{2}\right) \quad \text { with } \quad 0 \leq x_{2} \leq 1,
\end{aligned}
$$

where $\sqrt{s}$ is the hadronic c.m. energy, and $x_{1}$ and $x_{2}$ are the longitudinal fractions of the corresponding hadron momenta carried by each parton. Their ranges assure compatibility with the parton-level kinematics in the hadronic c.m. frame, where we impose that $m_{b} \leq p_{b}^{0} \leq$ $\frac{\sqrt{s}}{2}$ and $0 \leq p_{g}^{0} \leq \frac{\sqrt{s}}{2}$. Of course, the symmetric case in which $x_{1}$ and $x_{2}$ are exchanged is also considered, and, due to the massiveness of the initial-state $b$ quark, care must be taken to keep the two kinematic cases separate when convoluting the partonic cross section with the PDF to obtain the hadronic cross section. In the case of $b \bar{b} \rightarrow Z b \bar{b}$ with an initial-state massive $b$, the initial-state kinematic configuration becomes [37]

$$
\begin{aligned}
p_{b}= & \frac{\sqrt{s}}{2}\left(x_{1}+\frac{m_{b}^{2}}{x_{1} s}, 0,0, x_{1}-\frac{m_{b}^{2}}{x_{1} s}\right) \\
\text { and } \quad p_{\bar{b}}= & \frac{\sqrt{s}}{2}\left(x_{2}+\frac{m_{b}^{2}}{x_{2} s}, 0,0,-x_{2}+\frac{m_{b}^{2}}{x_{2} s}\right) \\
& \text { with } \quad \frac{m_{b}}{\sqrt{s}} \leq x_{1,2} \leq \frac{1}{2}\left(1+\sqrt{1-\frac{4 m_{b}^{2}}{s}}\right) .
\end{aligned}
$$

In the 5FS, which assumes a massless $b$ quark, the definition of the parton-level kinematics for $b g \rightarrow Z b$ is the same as for massless partons [obtained by setting $m_{b}=0$ in Eqs. (4) and (5)].

In the following we will review the essential features of the $O\left(\alpha_{s}\right)$ and $O(\alpha)$ virtual and real corrections in Secs. II A and II B, respectively, and discuss the choice of PDF subtraction terms in Sec. II C.

\section{A. Virtual corrections with NLOX}

The virtual $O\left(\alpha_{s}\right)$ and $O(\alpha)$ corrections to $\sigma_{\mathrm{LO}}$, contributing to $\sigma_{\mathrm{NLO}}^{\mathrm{QCD}}$ and $\sigma_{\mathrm{NLO}}^{\mathrm{EW}}$, respectively, have been produced with the one-loop provider NLOX [35], interfaced to a selection of in-house Monte Carlo integration routines. ${ }^{4}$ UV divergences are renormalized. For what pertains to IR divergences, the virtual IR poles are collected and their cancellation against the IR poles arising from the $O\left(\alpha_{s}\right)$ and $O(\alpha)$ real corrections, as well as from the corresponding PDF subtraction terms, is checked.

In Sec. II A 1 we will give a brief description of NLOX. More details will soon be available in Ref. [35], which will go along with a public release of the code as well as a more general interface to publicly available Monte Carlo integrators and event generators.

In Sec. II A 2 thereafter we will provide a description of our QCD renormalization, in order to make clear certain aspects that are also relevant for the discussion of our PDF subtraction terms in Sec. II C.

\section{NLOX}

NLOX is a new program for the automated computation of one-loop QCD and EW corrections in the Standard Model. A nonpublic predecessor of NLOX has been available in the past, to calculate one-loop QCD corrections to selected processes $[40,41]$. NLOX has seen quite some progress in recent years, and most recently partook in a technical comparison on NLO EW automation [42]. The current version of the program provides fully renormalized QCD and EW one-loop corrections in the Standard Model, for all the possible QCD + EW mixed coupling-power combinations to a certain parton-level process up to one-loop accuracy, including the full mass dependence on initialand final-state particle masses.

NLOX is based on a traditional approach of Feynman diagrams, utilizing QGRAF [43], FORM [44], and PYTHON to algebraically generate $\mathrm{C}++$ code for the virtual QCD and EW one-loop contributions to a certain process in terms of one-loop tensor-integral coefficients. The tensor-integral

\footnotetext{
${ }^{4}$ The $O(\alpha)$ and $O\left(\alpha_{s}\right)$ corrections at the amplitude-squared level have been provided by NLOX and cross-checked on the phase-space point level against several independent in-house codes and the one-loop provider Recola [38]. At the crosssection level NLOX and RECOLA have been used with a dedicated interface to the CUBA library [39], in order to confirm the virtual cross-section numbers from interfacing NLOX and the in-house codes to in-house Monte Carlo integration routines.
} 
coefficients are calculated recursively at runtime through standard reduction methods by the C++ library TRED, an integral part of NLOX. Several reduction techniques are available to TRED, many of which are found in Refs. $[45,46]$. The scalar one-loop integrals are evaluated by using either ONELOOP [47] or QCDLOOP $[48,49]$.

In NLOX UV and IR singularities are regularized in $d$-dimensional regularization (with $d=4-2 \epsilon,|\epsilon| \ll 1$ ). UV singularities are renormalized, while IR singularities are reported in terms of the Laurent coefficients of the corresponding $1 / \epsilon^{2}$ and $1 / \epsilon$ poles. The $U V$ renormalization in NLOX is carried out by means of counterterm diagrams, which provides a flexible way to systematically include mass renormalization for massive propagators as well as Yukawa-type vertices.

The renormalization constants in terms of which the EW UV counterterms are formulated are derived in the on-shell renormalization scheme, as described in Ref. [50], or in the complex-mass scheme, ${ }^{5}$ as described in Ref. [51]. As EW input scheme choices NLOX provides both the $\alpha(0)$ and the $G_{\mu}$ EW input schemes [50,52-54]. Per default the $\alpha(0)$ EW input scheme is used. The renormalization constants in terms of which the QCD UV counterterms are formulated are derived in a mixed renormalization scheme $[55,56]$ : the on-shell renormalization scheme is used for the wavefunction and mass renormalization of massive quarks, while the $\overline{\mathrm{MS}}$ renormalization scheme is used for massless quarks and gluons, where, however, in the latter case heavy-quark-loop contributions are decoupled by subtracting them at zero momentum. Since in this paper we present QCD results for both 5FS and m5FS, we briefly discuss in Sec. II A 2 the definition of those QCD counterterms that differ in the two schemes.

\section{Gluon wave-function and $\alpha_{s} Q C D$ renormalization: 5FS vs $\mathrm{m} 5 F S$}

As discussed in Sec. I, the interest in considering an initial-state massive $b$ quark and developing a m5FS formalism does not arise from the need of treating the $b$ quark as a heavy quark in the same way as the $t$ quark is (in which case a 4FS would be more appropriate), but from the intent of properly matching $b$-quark mass effects at the kinematic, hard matrix-element, and PDF level to reduce the theoretical uncertainty in processes involving $b$ quarks/ jets. The presence of a massive $b$ quark in our calculation mainly affects the IR structure of the $b$-loop contributions, but not the UV subtraction procedure where, in both the $5 \mathrm{FS}$ and the m5FS, the only quark treated as heavy (hence decoupled in the low-energy regime) is the $t$ quark. Indeed, in our calculation we renormalize the gluon two-point function by subtracting the contribution of all light quarks

\footnotetext{
${ }^{5}$ Note that for $Z+b$-jet production to work in the on-shell approximation for a real final-state $Z$ boson we neglect all widths, making all masses and derived parameters real quantities.
}

(including the $b$ quark) in $\overline{\mathrm{MS}}$ and the contribution of the $t$ quark at zero momentum, such that only the $t$ quark is decoupled in the low-energy limit. To be more explicit, our prescription corresponds to writing the renormalized transverse gluon self-energy as

$$
\begin{aligned}
\hat{\Sigma}_{G}^{T}\left(p^{2} ;\{q, Q\}\right)= & \left(\Sigma_{G}^{T}\left(p^{2} ;\{q, Q\}\right)-\delta Z_{G}(\{q, Q\})\right) \\
= & \left(\Sigma_{G}^{T}\left(p^{2} ;\{q\}\right)-\Sigma_{G}^{T}\left(p^{2} ;\{q\}\right) \mid \overline{\mathrm{MS}} \mathrm{UV} \text { pole }\right) \\
& +\left(\Sigma_{G}^{T}\left(p^{2} ;\{Q\}\right)-\Sigma_{G}^{T}(0 ;\{Q\})\right),
\end{aligned}
$$

where $\{q, Q\}$ is shorthand to denote the set $\{\{q\},\{Q\}\}$, formed by the sets $\{q\}$ (with dimension $n_{l f}$ ) and $\{Q\}$ (with dimension $n_{h f}$ ) of all quarks whose UV divergences from closed quark-loop contributions are subtracted in $\overline{\mathrm{MS}}$ or at zero momentum, respectively. In Eq. (6), $\Sigma_{G}^{T}\left(p^{2} ;\{q\}\right)$ contains the contributions from gluon and ghost loops, as well as from the $\{q\}$-quark loops, while $\Sigma_{G}^{T}\left(p^{2} ;\{Q\}\right)$ contains the contributions from the $\{Q\}$-quark loops, where in both the 5FS and the m5FS $\{Q\}=\{t\}$. This prescription defines the gluon-field renormalization constant $Z_{G}=1+\delta Z_{G}$, with $\delta Z_{G}$ given by

$$
\begin{aligned}
\delta Z_{G}(\{q, Q\})= & -\left.\Sigma_{G}^{T}\left(p^{2} ;\{q\}\right)\right|_{\overline{\mathrm{MS}} \mathrm{UV} \text { pole }}-\Sigma_{G}^{T}(0 ;\{Q\}) \\
= & -\frac{\alpha_{s}}{4 \pi} \frac{2}{3} S_{\epsilon}\left(\frac{1}{\epsilon_{\mathrm{uv}}}\left(\left(n_{l f}+n_{h f}\right) 2 T_{R}-\frac{5}{2} C_{A}\right)\right. \\
& \left.-\sum_{\{Q\}} 2 T_{R} \ln \left(\frac{m_{Q}^{2}}{\mu^{2}}\right)\right),
\end{aligned}
$$

and correspondingly the strong coupling renormalization constant $Z_{g_{s}}=1+\delta Z_{g_{s}}$, with $\delta Z_{g_{s}}$ given by

$$
\begin{aligned}
\delta Z_{g_{s}} & (\{q, Q\}) \\
= & -\frac{\alpha_{s}}{4 \pi} \frac{2}{3}\left(-\frac{1}{2}\right) S_{\epsilon}\left(\frac{1}{\epsilon_{\mathrm{uv}}}\left(\left(n_{l f}+n_{h f}\right) 2 T_{R}-\frac{11}{2} C_{A}\right)\right. \\
& \left.-\sum_{\{Q\}} 2 T_{R} \ln \left(\frac{m_{Q}^{2}}{\mu^{2}}\right)\right),
\end{aligned}
$$

where in Eqs. (7) and (8) we have made explicit that the poles in $\epsilon$ are of ultraviolet origin, $\mu$ is the 't Hooft mass in dimensional regularization, which is typically set equal to the renormalization scale $\mu_{r}, S_{\epsilon} \equiv(4 \pi)^{\epsilon} / \Gamma(1-\epsilon)$, and we have used $C_{A}=N_{c}=3, C_{F}=\left(N_{c}^{2}-1\right) /\left(2 N_{c}\right)$, and $T_{R}=1 / 2$. As a result in the m5FS the running of $\alpha_{s}$ is also governed by $n_{f}=5$ flavors, consistently with the set of PDF we choose to use (see Secs. II C and III). The only difference introduced in considering a massive $b$ quark arises in the IR parts of the renormalization counterterms, more specifically in the gluon-field residue, which, given the prescription we adopted for the gluon-field renormalization, is nontrivial and given by 


$$
\begin{aligned}
\bar{R}_{G}\left(\left\{q^{\prime}, Q^{\prime}\right\},\{q, Q\}\right) & =1-\Sigma_{G}^{T}\left(0 ;\left\{q^{\prime}, Q^{\prime}\right\}\right)-\delta Z_{G}(\{q, Q\}) \\
& =1+\delta \bar{R}_{G},
\end{aligned}
$$

where

$$
\begin{aligned}
\delta \bar{R}_{G}\left(\left\{q^{\prime}, Q^{\prime}\right\},\{q, Q\}\right) & \\
= & -\frac{\alpha_{s}}{4 \pi} \frac{2}{3}\left(-S_{\epsilon}\right)\left(\frac{1}{\epsilon_{\mathrm{ir}}}\left(n_{l f}^{\prime} 2 T_{R}-\frac{5}{2} C_{A}\right)\right. \\
& \left.+2 T_{R}\left(\sum_{\left\{Q^{\prime}\right\}} \ln \left(\frac{m_{Q^{\prime}}^{2}}{\mu^{2}}\right)-\sum_{\{Q\}} \ln \left(\frac{m_{Q}^{2}}{\mu^{2}}\right)\right)\right) .
\end{aligned}
$$

Notice that in Eq. (10) we have made explicit the fact that the remaining poles in $\epsilon$ are of infrared origin, and we have emphasized the difference between 5FS and m5FS by introducing a primed notation such that in the 5FS $\left\{Q^{\prime}\right\}=\{Q\}=\{t\}$ and $n_{l f}^{\prime}=n_{l f}=5$, while in the m5FS $\left\{Q^{\prime}\right\}=\{b, t\},\{Q\}=\{t\}, n_{l f}^{\prime}=4$, and $n_{l f}=5$.

\section{B. Real corrections}

The $O\left(\alpha_{s}\right)$ real corrections to $b g \rightarrow Z b$ contain both soft and collinear singularities. On top of gluon emission from $b g \rightarrow Z b \quad(b g \rightarrow Z b+g)$, they also include the $q b(\bar{q} b) \rightarrow Z b+q(\bar{q}), g g \rightarrow Z b \bar{b}, q \bar{q} \rightarrow Z b \bar{b}$ (with $q=$ $u, d, s, c)$, and $b \bar{b} \rightarrow Z b \bar{b}$ channels. To separate the singular regions of phase space and extract analytically the corresponding IR singularities, we used a phase-spaceslicing (PSS) method with both soft $\left(\delta_{s}\right)$ and collinear $\left(\delta_{c}\right)$ cutoffs, in terms of which the emission of a gluon with fourmomentum $k$ is defined by the condition $k^{0}<\delta_{s} \sqrt{\hat{s}} / 2$ if the gluon is soft, or by the condition $p_{i} k<\delta_{c} k^{0} p_{i}^{0}$ if the gluon is collinear to another massless parton with fourmomentum $p_{i}$ (where the momenta are defined in the parton-level c.m. frame with c.m. energy $\sqrt{\hat{s}}$ ). The procedure is well known and comprehensively summarized in Ref. [57] for both the case of initial-state and final-state singularities. In particular, for the phase-space integrals originating from a soft emitted parton we used the method and expressions reviewed in Ref. [50]. Results have been cross-checked with two independent codes.

The $O(\alpha)$ real corrections to $b g \rightarrow Z b$ consist of both photon $(\gamma)$ and EW gauge and Higgs boson emissions. The cross sections for real $\mathrm{Z} / \mathrm{W} / \mathrm{H}$ emission are finite since their masses provide a physical IR cutoff and thus can be considered separately. These processes have very distinct signatures and their inclusion in the evaluation of the inclusive cross section for $Z+b$-jet production depends on the experimental signature selected. An example of a set of analysis cuts in $Z+j$ production which warrants the inclusion of real EW gauge-boson emission in addition to virtual EW corrections can be found in Ref. [58], where the well-known incomplete cancellation of EW Sudakov logarithms between these two contributions $[59,60]$ is also discussed. Since our study is not aiming at a detailed analysis of this effect, which should only be done in collaboration with the experimentalists performing the measurement of $Z+b$-jet production, we do not consider the real emission of an extra $Z / W / H$. On the other hand, we consider the QED part of the real radiation (photon emission) which needs to be consistently included in order to cancel the IR divergences present in the virtual cross section (due to photon exchange).

The calculation of the $O(\alpha)$ real-photon emission cross section can easily be implemented using PSS [57], and actually involves just a subset of the singularities encountered in the QCD case for massive $b$ quarks. With due differences, the result can easily be obtained from there. On the other hand, the QED case provides an interesting testing ground for the implementation of the dipole subtraction (DS) method with initial-state massive dipoles, whose working knowledge, in the QCD as well as QED cases, is a necessary step toward the proper implementation of processes with initial-state massive partons in NLO event generators. All dipoles for QED radiation off leptons, including initial-state massive dipoles, have been calculated in [36], where they have also been tested against phasespace slicing in specific QED processes. QED massive dipoles have also been used and tested against PSS in [61] for hadronic $W+$ jet production. In the specific case of photon emission from the $b$-quark lines of $b g \rightarrow Z b$ we have implemented the dipole subtraction terms corresponding to the configurations of massive initial-state emitter and massive final-state spectator (both $b$ quarks), and vice versa. For illustration, a summary of the comparison between the two methods is given in Table I, where we report the total NLO EW cross section calculated using either PSS or DS. In the case of PSS the result depends on the soft cutoff $\delta_{s}$, and we report in Table I the values of $\sigma_{\mathrm{NLO}}^{\mathrm{EW}}$ for four decreasing values of $\delta_{s}$, to show the existence of a plateau region in which $\sigma_{\mathrm{NLO}}^{\mathrm{EW}}$ is actually independent of $\delta_{s}$. We notice that the results reported in Table I have been obtained without imposing a recombination cut, and therefore slightly differ from what is included in the final results presented in Sec. III. Indeed, Table I shows that results obtained using the PSS method with a soft cutoff $\delta_{s}$ varying between $10^{-3}$ and $10^{-5}$ are compatible with results

TABLE I. Comparison of the NLO EW cross section obtained with the PSS and DS method for $Z+b$-jet production at the $13 \mathrm{TeV}$ LHC in the setup described in Sec. III. See the text for more details.

\begin{tabular}{lccc}
\hline \hline$\sigma_{\mathrm{LO}}[\mathrm{pb}]$ & Method & $\delta_{s}$ & $\sigma_{\mathrm{NLO}}^{\mathrm{EW}}[\mathrm{pb}]$ \\
\hline $389.727 \pm 0.005$ & Phase-space slicing & $10^{-2}$ & $383.342 \pm 0.005$ \\
& & $10^{-3}$ & $383.344 \pm 0.005$ \\
& & $10^{-4}$ & $383.345 \pm 0.006$ \\
& & $10^{-5}$ & $383.346 \pm 0.006$ \\
& & & $383.336 \pm 0.006$ \\
\hline \hline
\end{tabular}


obtained using the DS method based on Ref. [36], to which we refer for more technical details. If the successful implementation of the PSS method depends on a careful study of the analytical dependence on the soft and collinear cutoffs, obtaining meaningful numerical results using the dipole method involves its own subtleties. Hence, the crosscheck between the two methods is all but trivial and allows us to move forward to further extensions and future studies with more confidence. We notice that initial-state massive QCD dipoles have first been studied in Ref. [62], where, however, very little detail is provided, while a more detailed discussion has recently appeared in Ref. [34].

\section{PDF subtraction terms}

After combining real and virtual NLO QCD or NLO EW corrections, residual poles in dimensional regularization, due to collinear radiation off massless initial-state partons, are absorbed into NLO PDF via $\overline{\mathrm{MS}}$ factorization. This is systematically achieved by defining PDF subtraction terms, also known as collinear counterterms.

The corresponding treatment in the case of massive initial-state quarks needs a more detailed discussion. Indeed, in both NLO QCD and NLO EW calculations with a massive $b$ quark, the $b$-quark mass acts as a physical regulator for collinear singularities in $b \rightarrow b(g, \gamma)$ and $(g, \gamma) \rightarrow b \bar{b}$ splittings. In particular, radiative corrections involving a massive initial-state $b$ quark do not contain collinear poles in dimensional regularization, but logarithms of the $b$-quark mass instead, which, being finite, do not necessarily need to be absorbed into the $b$-quark PDF. However, since at LHC energies the mass of the $b$ quark is relatively small, these mass logarithms can be large and retaining them in the calculation of the partonic cross section, i.e., not absorbing them into the $b$-quark PDF, can lead to unnaturally large corrections that could eventually affect the numerical stability of the hadronic cross section. In a fully massive calculation the numerical stability of the hadronic cross section is retained by absorbing these mass logarithms via, e.g., the generalized $\overline{\mathrm{MS}}$ scheme of Collins, also known as the ACOT scheme [31,32] (see also Ref. [63]). ${ }^{6}$ This is achieved by defining corresponding PDF subtraction terms in the case of near-collinear emission off massive $b$ quarks, quite in analogy to subtracting poles in dimensional regularization in the case of collinear emission off massless $b$ quarks, such that $\overline{\mathrm{MS}}$ factorization is retained in the massless limit.

\footnotetext{
${ }^{6}$ For an overview on the ACOT scheme, as well as its applicability over a range of scales and its convergence to the $\overline{\mathrm{MS}}$ scheme in the massless limit, see, e.g., Refs. [53,63-65]. For a review and a comparison of the heavy-quark schemes based on ACOT, TR, and FONLL used, respectively, in the CTEQ, MSTW (now MMHT), and NNPDF PDF sets, see, e.g., Ref. [66]. A thorough discussion of the case of charm-quark initiated processes in the FONLL scheme can be found for instance in Refs. [67-69].
}

In obtaining the 5FS NLO QCD results presented in Sec. III we have used the $S$-ACOT scheme (or simplified ACOT scheme) [33], where the $b$ quark is considered to be massless everywhere, except in the calculation of those partonic subprocesses that only involve $b$ quarks in the final state. ${ }^{7}$ As such, in this scheme the $b$-quark mass is used as a regulator for the $g \rightarrow b \bar{b}$ splitting, while the initial-state $b \rightarrow b g$ splitting is treated as for all massless quarks (namely, initial-state collinear poles are absorbed in the corresponding PDF, by PDF subtraction terms, in $\overline{\mathrm{MS}}$ factorization). The S-ACOT scheme is what is usually assumed in implementing a traditional 5FS (as also done in Ref. [20]), and is also what is assumed in defining the corresponding PDF in recent CTEQ PDF sets, including the CT14QED PDF set that we use for our study. ${ }^{8}$

On the other hand, in obtaining NLO QCD and NLO EW results in the m5FS we need to implement a fully massive factorization scheme such as the ACOT scheme, where the $b$ quark is considered massive everywhere. In this case, mass logarithms originating from both $g \rightarrow b \bar{b}$ (or $\gamma \rightarrow b \bar{b}$, in case initial-state photons are considered) and $b \rightarrow b g$ (or $b \rightarrow b \gamma$ ) splittings are absorbed into the $b$-quark PDF, and all partonic subprocesses are calculated considering a massive $b$ quark. ${ }^{9}$ In accordance with this, in our m5FS calculation of $O(\alpha)$ and $O\left(\alpha_{s}\right)$ corrections to $b g \rightarrow Z b$, we subtract from the partonic cross section all the mass logarithms arising from both initial-state $g \rightarrow b \bar{b}$ and $b \rightarrow b g$ (or $b \rightarrow b \gamma$ ) splittings [see Eqs. (12) and (14)]. In our study we use the $b$-quark PDF of the CT14QED PDF set [70]. For the best accuracy, however, a dedicated PDF set should be determined in the ACOT scheme, which is beyond the scope of this study. ${ }^{10}$

As a reminder on how the PDF subtraction terms enter our calculation, in a condensed notation, the NLO cross section, using a PSS method, can be sketched as follows:

$$
\begin{aligned}
\sigma= & \sigma_{\mathrm{LO}}+\sigma_{V}+\sigma_{R}+\sigma_{\text {sub }} \\
= & \sigma_{\mathrm{LO}}+\sigma_{V}+\left(\sigma_{\text {soft } / \text { coll }}\left(\left\{\delta_{s}, \delta_{c}\right\}\right)+\sigma_{\text {hard } / \text { non-coll }}\left(\left\{\delta_{s}, \delta_{c}\right\}\right)\right) \\
& +\sigma_{\text {sub }},
\end{aligned}
$$

\footnotetext{
${ }^{7}$ As a consequence, both $g g \rightarrow Z b \bar{b}$ and $q \bar{q} \rightarrow Z b \bar{b}$ have been calculated with a massive $b$ quark in both the 5FS and the m5FS cases.

${ }^{8}$ The CT14QED PDF set [70] is determined in the same CTEQ global analysis in which the CT14 PDF set [71] is determined, using the S-ACOT $(\chi)$ scheme [64], but including QED in the DGLAP evolution of the PDF.

${ }^{9}$ Although one could use the S-ACOT scheme, which agrees with the ACOT scheme in the high-energy limit, for best accuracy and a fully consistent treatment of massive initial-state quarks, including the possibility of constraining an intrinsic $b$-quark parton density, the use of a scheme like the ACOT scheme should be preferred. For an overview on ACOT vs S-ACOT see, e.g., Refs. [53,63-65].

${ }^{10}$ The study presented in Ref. [34] pursues the direction of determining dedicated PDF sets in a fully massive scheme, also focusing on the definition of a m5FS.
} 
where $\sigma_{V}$ denotes the set of UV renormalized virtual contributions and $\sigma_{R}$ the set of real-emission contributions. In the real-emission contributions, soft and collinear photon/parton emissions are treated using a PSS method with a soft $\left(\delta_{s}\right)$ and a collinear $\left(\delta_{c}\right)$ cutoff, as defined at the beginning of Sec. II B, separating the real-emission contributions into soft/collinear $\left(\sigma_{\text {soft } / \text { coll }}\right)$ and hard/noncollinear $\left(\sigma_{\text {hard/non-coll }}\right)$ contributions. In the following, we will collect the expressions of those PDF subtraction terms $\left(\sigma_{\text {sub }}\right)$, which are modified by the presence of a massive $b$ quark or by adopting a m5FS versus a 5FS. Details about the results reported in these sections can be found in the literature (see, e.g., Refs. [32,33,53,57,63,65,72-74]). We will keep explicit renormalization $\left(\mu_{r}\right)$ and factorization $\left(\mu_{f}\right)$ dependence, but we will not distinguish between QED and QCD factorization scales.

As discussed above, in both the 5FS and the m5FS schemes, the $b$-quark mass appears as a physical regulator of the collinear singularity in the $g \rightarrow b \bar{b}$ splitting, and the corresponding subtraction term reads $[32,63,65]$

$$
\begin{aligned}
\sigma_{\text {sub }}^{g \rightarrow b \bar{b}}= & -\int d x_{1} d x_{2} \frac{\alpha_{s}\left(\mu_{r}\right)}{2 \pi}\left\{\int_{x_{1}}^{1} \frac{d z}{z} g\left(\frac{x_{1}}{z}, \mu_{f}\right) g\left(x_{2}, \mu_{f}\right)\right. \\
& \times \frac{1}{2}\left[z^{2}+(1-z)^{2}\right] \ln \left(\frac{\mu_{f}^{2}}{m_{b}^{2}}\right) \hat{\sigma}_{\mathrm{LO}} \\
& \left.+\left(x_{1} \leftrightarrow x_{2}\right)\right\}
\end{aligned}
$$

where $\hat{\sigma}_{\mathrm{LO}}=\hat{\sigma}_{\mathrm{LO}}\left(x_{1}, x_{2}, \mu_{r}\right)$ denotes the tree-level partonic cross section for $b g \rightarrow Z b$ (calculated with a massless $b$ quark in the 5FS and with a massive $b$ quark in the m5FS), and the last line takes into account that an analogous term where the role of the two gluons is exchanged needs to be included. Here and in the following it is also understood that the analogous subtraction terms for $\bar{b} g \rightarrow Z \bar{b}$ are considered.

In contrast, the collinear $b \rightarrow b g$ (and $b \rightarrow b \gamma$ ) initialstate splittings occurring in the $O\left(\alpha_{s}\right)$ [and $O(\alpha)$ ] corrections to the $b g$-initiated processes are treated quite differently in the traditional 5FS and a m5FS. In a 5FS NLO calculation (with a massless initial-state $b$ quark) the corresponding PDF subtraction term reads [57]

$$
\begin{aligned}
\sigma_{\mathrm{sub}}^{b \rightarrow b(\gamma, g)}= & -S_{\epsilon}\left(\frac{\mu_{r}^{2}}{\mu_{f}^{2}}\right)^{\epsilon} \int d x_{1} d x_{2}\left\{\frac{\alpha_{i}}{2 \pi} C_{i}\right. \\
& \times\left\{\int_{x_{1}}^{1-\delta_{s}} \frac{d z}{z} b\left(\frac{x_{1}}{z}, \mu_{f}\right) g\left(x_{2}, \mu_{f}\right)\left(\frac{1+z^{2}}{1-z}\right)\left(-\frac{1}{\epsilon}\right)\right. \\
& \left.+b\left(x_{1}, \mu_{f}\right) g\left(x_{2}, \mu_{f}\right)\left[-\frac{1}{\epsilon}\left(2 \ln \delta_{s}+\frac{3}{2}\right)\right]\right\} \hat{\sigma}_{\mathrm{LO}} \\
& \left.+\left(x_{1} \leftrightarrow x_{2}\right)\right\},
\end{aligned}
$$

where $\alpha_{i}=\alpha$ and $C_{i}=q_{b}^{2}$ (with $q_{b}$ the charge of the $b$ quark) for $O(\alpha)$ EW corrections, while $\alpha_{i}=\alpha_{s}$ and $C_{i}=$ $C_{F}=\left(N_{c}^{2}-1\right) / 2 / N_{c}$ (with $N_{c}=3$ ) for $O\left(\alpha_{s}\right)$ QCD corrections. Here and in the following the last line takes into account that an analogous term where $b$ and $g$ come from the opposite, respective hadrons needs to be included. Note that, performing the $d z$ integration in Eq. (13), only subleading terms in $\delta_{s}$ remain, which vanish in the limit $\delta_{s} \rightarrow 0$ [this is also true for Eqs. (14), (15), and (16) in the remainder of this section]. On the other hand, in the m5FS (with a massive initial-state $b$ quark) the PDF subtraction term reads $[53,63,65,72-74]$

$$
\begin{aligned}
\sigma_{\text {sub }}^{b \rightarrow b(\gamma, g)}= & -\int d x_{1} d x_{2}\left\{\frac { \alpha _ { i } } { 2 \pi } C _ { i } \left\{\int_{x_{1}}^{1-\delta_{s}} \frac{d z}{z} b\left(\frac{x_{1}}{z}, \mu_{f}\right)\right.\right. \\
& \times g\left(x_{2}, \mu_{f}\right)\left(\frac{1+z^{2}}{1-z}\right)\left[\ln \left(\frac{\mu_{f}^{2}}{m_{b}^{2}} \frac{1}{(1-z)^{2}}\right)-1\right] \\
& +b\left(x_{1}, \mu_{f}\right) g\left(x_{2}, \mu_{f}\right)\left[\ln \left(\frac{\mu_{f}^{2}}{m_{b}^{2}}\right)\left(2 \ln \delta_{s}+\frac{3}{2}\right)\right. \\
& \left.\left.-2 \ln \delta_{s}-2 \ln ^{2} \delta_{s}+2\right]\right\} \hat{\sigma}_{\mathrm{LO}} \\
& \left.+\left(x_{1} \leftrightarrow x_{2}\right)\right\} .
\end{aligned}
$$

Finally, in the m5FS NLO QCD calculation the subtraction term for the $g \rightarrow g g$ splitting is also affected by the $b$-quark mass as follows [74]:

$$
\begin{aligned}
\sigma_{\mathrm{sub}}^{g \rightarrow g g}= & -S_{\epsilon}\left(\frac{\mu_{r}^{2}}{\mu_{f}^{2}}\right)^{\epsilon} \int d x_{1} d x_{2}\left\{\frac{\alpha_{s}\left(\mu_{r}\right)}{2 \pi} C_{g}\right. \\
& \times\left\{\int_{x_{2}}^{1-\delta_{s}} \frac{d z}{z} b\left(x_{1}, \mu_{f}\right) g\left(\frac{x_{2}}{z}, \mu_{f}\right)\right. \\
& \times\left[\frac{z}{1-z}+\frac{1-z}{z}+z(1-z)\right]\left(-\frac{2}{\epsilon}\right) \\
& +b\left(x_{1}, \mu_{f}\right) g\left(x_{2}, \mu_{f}\right)\left[-\frac{1}{\epsilon}\left(2 \ln \delta_{s}+\frac{11}{6}-\frac{n_{l f^{\prime}}}{3 C_{g}}\right)\right. \\
& \left.\left.\left.-\frac{1}{9} \ln \left(\frac{\mu_{f}^{2}}{m_{b}^{2}}\right)\right]\right\} \hat{\sigma}_{\mathrm{LO}}+\left(x_{1} \leftrightarrow x_{2}\right)\right\},
\end{aligned}
$$

where $C_{g}=C_{A}=N_{c}$ (with $N_{c}=3$ ), and $n_{l f^{\prime}}=4$ (see also Sec. II A 2), whereas in the 5FS the same subtraction term reduces to [57] 


$$
\begin{aligned}
\sigma_{\mathrm{sub}}^{g \rightarrow g g}= & -S_{\epsilon}\left(\frac{\mu_{r}^{2}}{\mu_{f}^{2}}\right)^{\epsilon} \int d x_{1} d x_{2}\left\{\frac{\alpha_{s}\left(\mu_{r}\right)}{2 \pi} C_{g}\right. \\
& \times\left\{\int_{x_{2}}^{1-\delta_{s}} \frac{d z}{z} b\left(x_{1}, \mu_{f}\right) g\left(\frac{x_{2}}{z}, \mu_{f}\right)\right. \\
& \times\left[\frac{z}{1-z}+\frac{1-z}{z}+z(1-z)\right]\left(-\frac{2}{\epsilon}\right) \\
& \left.+b\left(x_{1}, \mu_{f}\right) g\left(x_{2}, \mu_{f}\right)\left[-\frac{1}{\epsilon}\left(2 \ln \delta_{s}+\frac{11}{6}-\frac{n_{l f^{\prime}}}{3 C_{g}}\right)\right]\right\} \hat{\sigma}_{\mathrm{LO}} \\
& \left.+\left(x_{1} \leftrightarrow x_{2}\right)\right\},
\end{aligned}
$$

with $n_{l f^{\prime}}=5$.

\section{NUMERICAL RESULTS}

In this section we present results for total cross sections and distributions of inclusive $Z+b$-jet production at the LHC with c.m. energy $13 \mathrm{TeV}$. As explained in Secs. I and II, we work in the m5FS and include the first order of QCD and EW corrections. We assess their relative impact, and the impact of considering $b$-quark mass effects by comparing NLO QCD results in the 5FS and the m5FS. The values for the SM input parameters are chosen as follows [75]:

$$
\begin{aligned}
G_{\mu} & =1.1663787 \times 10^{-5} \mathrm{GeV}^{-2}, \\
\alpha(0) & =1 / 137.035999074, \quad \alpha_{s} \equiv \alpha_{s}\left(M_{Z}^{2}\right)=0.118, \\
M_{Z} & =91.1876 \mathrm{GeV}, \\
M_{W} & =80.385 \mathrm{GeV}, \quad M_{H}=125 \mathrm{GeV}, \\
m_{e} & =0.510998928 \mathrm{MeV}, \quad m_{\mu}=0.1056583715 \mathrm{GeV}, \\
m_{\tau} & =1.77682 \mathrm{GeV}, \quad m_{c}=1.275 \mathrm{GeV}, \quad m_{t}=173 \mathrm{GeV}, \\
m_{u} & =0.06983 \mathrm{GeV}, \quad m_{c} \\
m_{d} & =0.06983 \mathrm{GeV}, \quad m_{s}=0.15 \mathrm{GeV}, \quad m_{b}=4.75 \mathrm{GeV} .
\end{aligned}
$$

The weak mixing angle is calculated from the weak gaugeboson masses, i.e., $\sin ^{2} \theta_{w}=1-M_{W}^{2} / M_{Z}^{2}$. The lepton and light-quark masses are only used in the evaluation of the renormalization constant for the electric charge, $\delta Z_{e}$, resulting in large logarithmic corrections. Such large universal EW corrections can be absorbed into the corresponding lowest-order cross sections by using specific EW input schemes, i.e., the $\alpha\left(M_{Z}\right)$ scheme and the $G_{\mu}$ scheme as described, e.g., in [50,52-54]. Here we provide results in the so-called $G_{\mu}$ scheme according to which in the Bornlevel couplings $\alpha(0)$ is replaced by $\alpha_{G_{\mu}}$,

$$
\alpha(0) \rightarrow \alpha_{G_{\mu}}=\frac{\sqrt{2} G_{\mu} M_{W}^{2}}{\pi}\left(1-\frac{M_{W}^{2}}{M_{Z}^{2}}\right),
$$

and $\delta Z_{e}$ receives a contribution from $\Delta r$ [76], which describes the EW one-loop corrections to muon decay. For the input parameters given in Eq. (17) we find $\Delta r=0.02968$. Consequently, the NLO EW cross section in the $G_{\mu}$ scheme is related to the NLO EW cross section in the $\alpha(0)$ scheme as follows:

$$
\sigma_{\mathrm{NLO}}^{\mathrm{EW}, G_{\mu}}=\left(\sigma_{\mathrm{NLO}}^{\mathrm{EW}, \alpha_{0}}-\Delta r \alpha(0) \alpha_{s} \sigma_{\mathrm{LO}}^{(1,1)}\right) \frac{\alpha_{G_{\mu}}}{\alpha(0)} .
$$

In this way, the large logarithmic dependence on the lepton and light quark masses due to $\delta Z_{e}$ in the EW one-loop corrections is completely canceled by the corresponding contribution in $\Delta r$, while $\mathrm{EW}$ universal corrections are included and resummed in the Born cross section. Note that we choose the relative EW corrections to be evaluated with $\alpha(0)$; i.e., in the virtual and real EW corrections $\alpha(0)^{2}$ is replaced by $\alpha(0) \alpha_{G_{\mu}}$, which is appropriate for photonic corrections. Alternatively, one can evaluate the relative corrections using $\alpha_{G_{\mu}}$, which differs from our choice by higher-order corrections.

Since we include both QCD and QED corrections, we choose to use the CT14QED PDF set [70] with $\alpha_{S}\left(M_{Z}\right)=0.118$. We do not include in our study an estimate of the current PDF uncertainty, since, for $b$-quark initiated processes, this will become more meaningful when a complete implementation of the m5FS is included in the available PDF sets. Likewise, we do not consider uncertainties from varying the $b$-quark mass. On the other hand, the choice of a PDF set which includes QED radiation is necessary when considering EW corrections. In both NLO and LO results we use NLO PDF and the twoloop running of $\alpha_{s}$ with $n_{l f}=5$ flavors [cf. Eq. (8)]. We choose to quantify the uncertainty from scale variation by varying $\mu_{r}$ by a factor of 2 above and below the central value $\mu_{r}=M_{Z}$, while keeping $\mu_{f}$ fixed at $\mu_{f}=M_{Z}$. Our choice is motivated by the fact that most of the residual scale dependence is driven by the NLO QCD cross section which varies monotonically with $\mu_{r}$ and $\mu_{f}$ in opposite directions [20]. It is therefore more appropriate not to vary the two scales together setting $\mu_{r}=\mu_{f}$. At the same time, varying both scales simultaneously and independently is not necessarily a better estimate of the theoretical uncertainty, which to be more accurate would require a dedicated study of both fixed and dynamical scale choices as well as other factors, such as the choice of different PDF sets. We notice, however, that a study of the $p_{T}$ distributions of leading and subleading $b$ jets in $p p \rightarrow Z b \bar{b}$ including NLO QCD corrections [25] seems to favor a choice of $\mu_{f}$ anywhere between 50 and $100 \mathrm{GeV}$ (where the typical $d p_{T} / p_{T}$ rescaling responsible for the leading part of the integrated bottom PDF is more evident). Hence our choice of $\mu_{f}=M_{Z}$. The bands presented in Figs. 3-7 have been built using this prescription. 

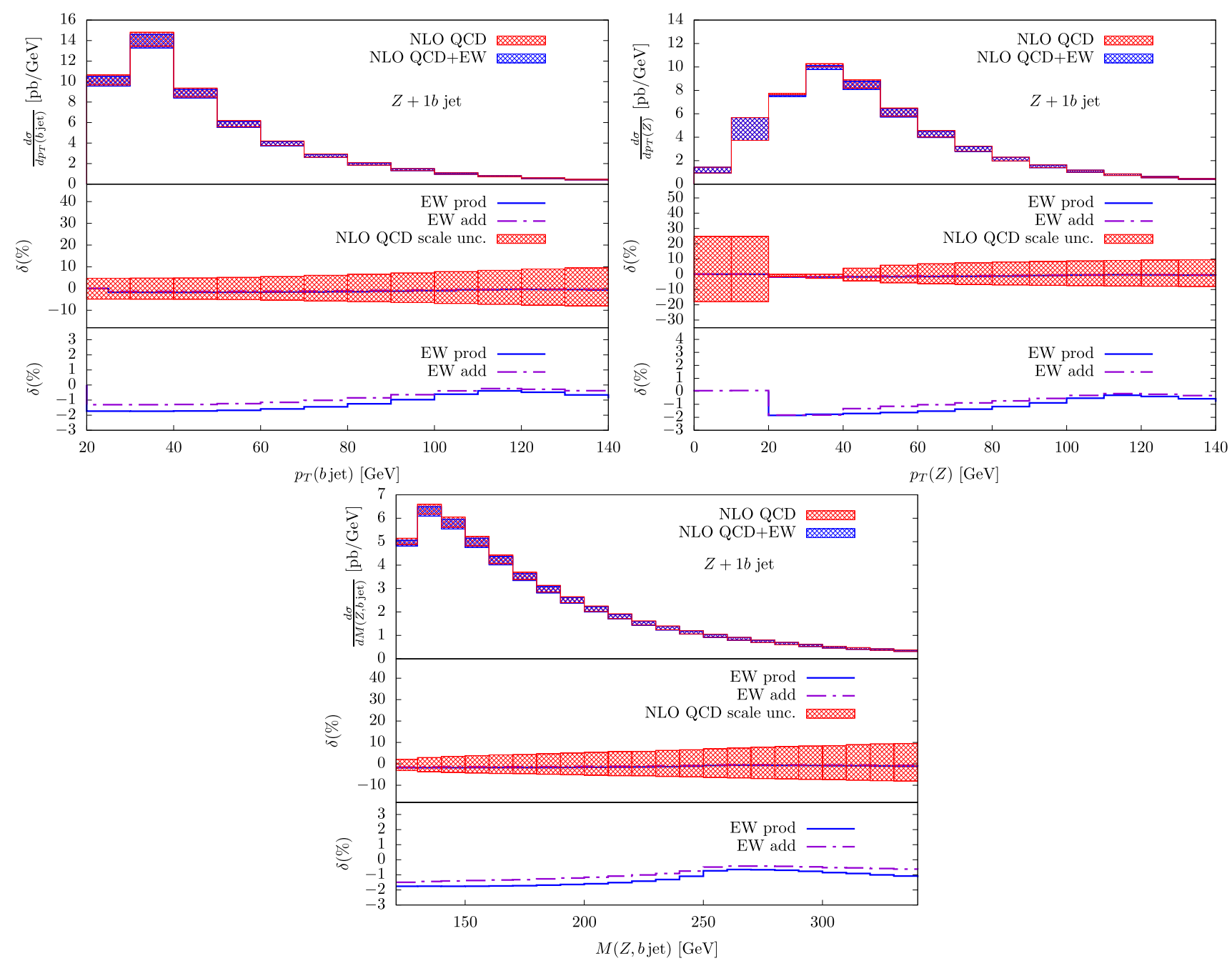

FIG. 3. Differential distributions for the $b$-jet and $Z$ transverse momentum, and the invariant mass of the $Z$ boson and the $b$ jet. The lower plots show the relative EW $O(\alpha)$ corrections differential distributions, $\delta_{\mathrm{EW}}^{\text {prod }}$ and $\delta_{\mathrm{EW}}^{\text {add }}$, together with the NLO QCD scale uncertainty in the middle plot.
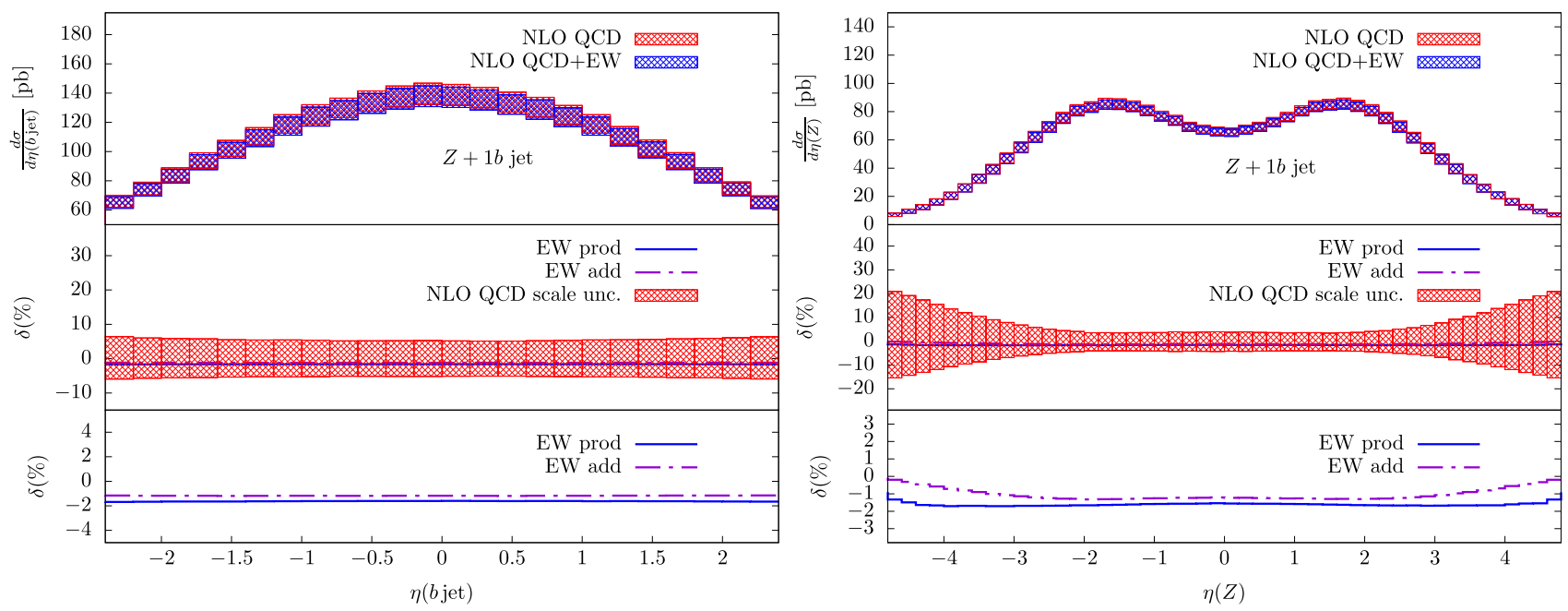

FIG. 4. Differential distributions for the $Z$ boson and $b$-jet pseudorapidity. The lower plots show the relative EW $O(\alpha)$ corrections differential distributions, $\delta_{\mathrm{EW}}^{\mathrm{prod}}$ and $\delta_{\mathrm{EW}}^{\text {add }}$, together with the NLO QCD scale uncertainty in the middle plot. 


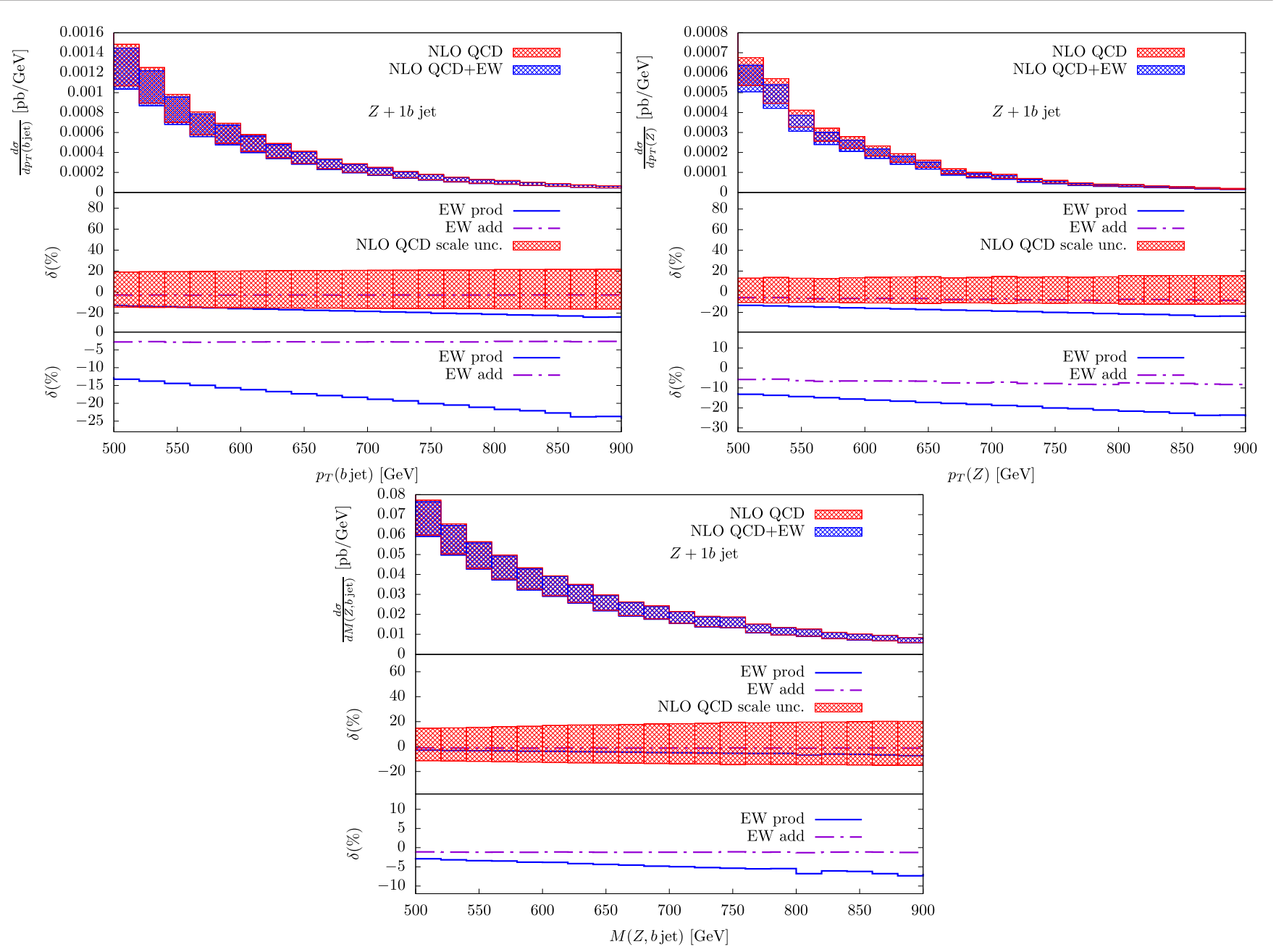

FIG. 5. Differential distributions for the $b$-jet and $Z$ transverse momentum, and the invariant mass of the $Z$ boson and the $b$ jet at high $p_{T}$ and high invariant $Z b$-jet mass. The lower plots show the relative EW $O(\alpha)$ corrections differential distributions, $\delta_{\mathrm{EW}}^{\text {prod }}$ and $\delta_{\mathrm{EW}}^{\text {add }}$, together with the NLO QCD scale uncertainty in the middle plot.

Since we consider inclusive $Z+b$-jet production, we include $Z+b+X$ and $Z+\bar{b}+X$ final states, with $X=\{$ light parton, $\gamma, b($ or $\bar{b})\}$, in the real-emission case. As we have at most two partons in the final state, we use a simple jet (recombination) algorithm; i.e., we recombine the final-state $b$ (or $\bar{b}$ ) quark with a final-state light quark, gluon, photon, $\bar{b}$ (or $b$ ) quark (for $Z b \bar{b}$ events), if their separation in the azimuthal angle-pseudorapidity plane, $\Delta R(b, X)=\sqrt{\left(\Phi_{b}-\Phi_{X}\right)^{2}+\left(\eta_{b}-\eta_{X}\right)^{2}}$, is less than $R_{\text {min }}=0.4$, as would be the case for any cone algorithm of this size. Moreover, we impose the following acceptance cuts on the transverse momentum and pseudorapidity of all $b$ jets: $p_{T}(b)>25 \mathrm{GeV}$ and $|\eta(b)|<2.5$, and we keep all events that have at least one $b$ jet. For events with $2 b$ jets, the differential distributions for $b$-jet observables show the hardest $b$ jet.

In Table II we present results for the total cross sections. In order to illustrate the impact of different orders of corrections on the total cross section we give results for both LO and higher-order cross sections, and we distinguish between the NLO cross sections obtained by including only QCD corrections $\left(\sigma_{\mathrm{NLO}}^{\mathrm{QCD}}\right)$, only EW corrections $\left(\sigma_{\mathrm{NLO}}^{\mathrm{EW}}\right)$, or both. We have combined NLO QCD and EW corrections according to both the additive $\left(\sigma_{\mathrm{NLO}}^{\mathrm{QCD}+\mathrm{EW})}\right)$ and multiplicative $\left(\sigma_{\mathrm{NLO}}^{\mathrm{QCD} \times \mathrm{EW})}\right)$ approaches. While in the additive approach the two sets of corrections are simply added [cf. Eq. (3)], i.e.,

$$
\sigma_{\mathrm{NLO}}^{\mathrm{QCD}+\mathrm{EW}}=\sigma_{\mathrm{LO}}+\alpha_{s}^{2} \alpha \sigma^{(2,1)}+\alpha_{s} \alpha^{2} \sigma^{(1,2)},
$$

while in the multiplicative approach the NLO QCD result is multiplied by the EW $K$ factor $\left(K_{\mathrm{EW}}\right)$ [77],

$$
\sigma_{\mathrm{NLO}}^{\mathrm{QCD} \times \mathrm{EW}}=\sigma_{\mathrm{NLO}}^{\mathrm{QCD}} \times \frac{\sigma_{\mathrm{NLO}}^{\mathrm{EW}}}{\sigma_{\mathrm{LO}}} \equiv \sigma_{\mathrm{NLO}}^{\mathrm{QCD}} \times K_{\mathrm{EW}},
$$

hence including mixed QCD $\times \mathrm{EW}$ corrections that are of higher order. As a first estimate of the overall effect of each 

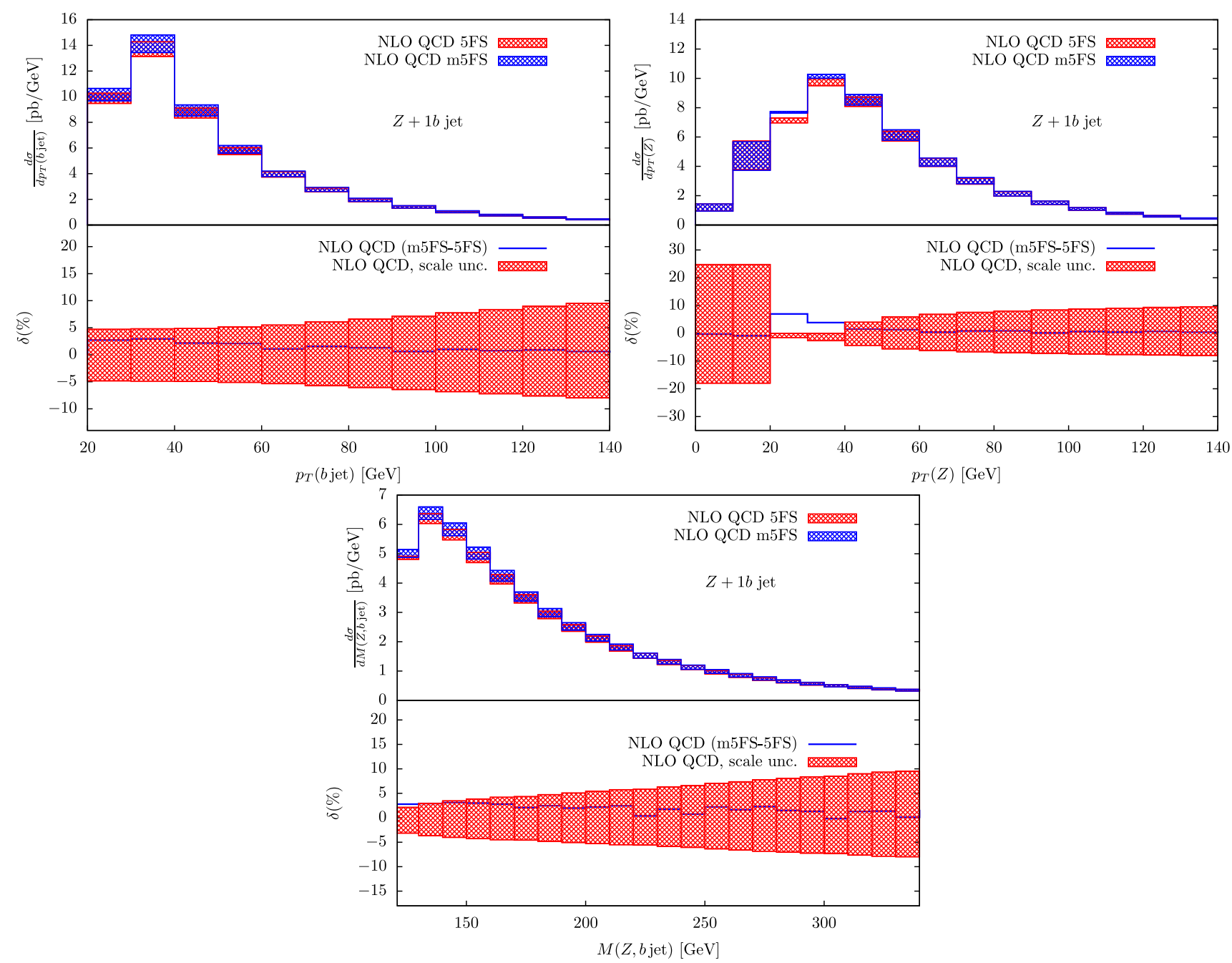

FIG. 6. The 5FS and m5FS NLO QCD predictions for differential distributions for the $b$-jet and $Z$ transverse momentum, and the invariant $Z b$-jet mass. The lower plot shows the difference $\delta_{\mathrm{mb}}$ together with the NLO QCD scale uncertainty.
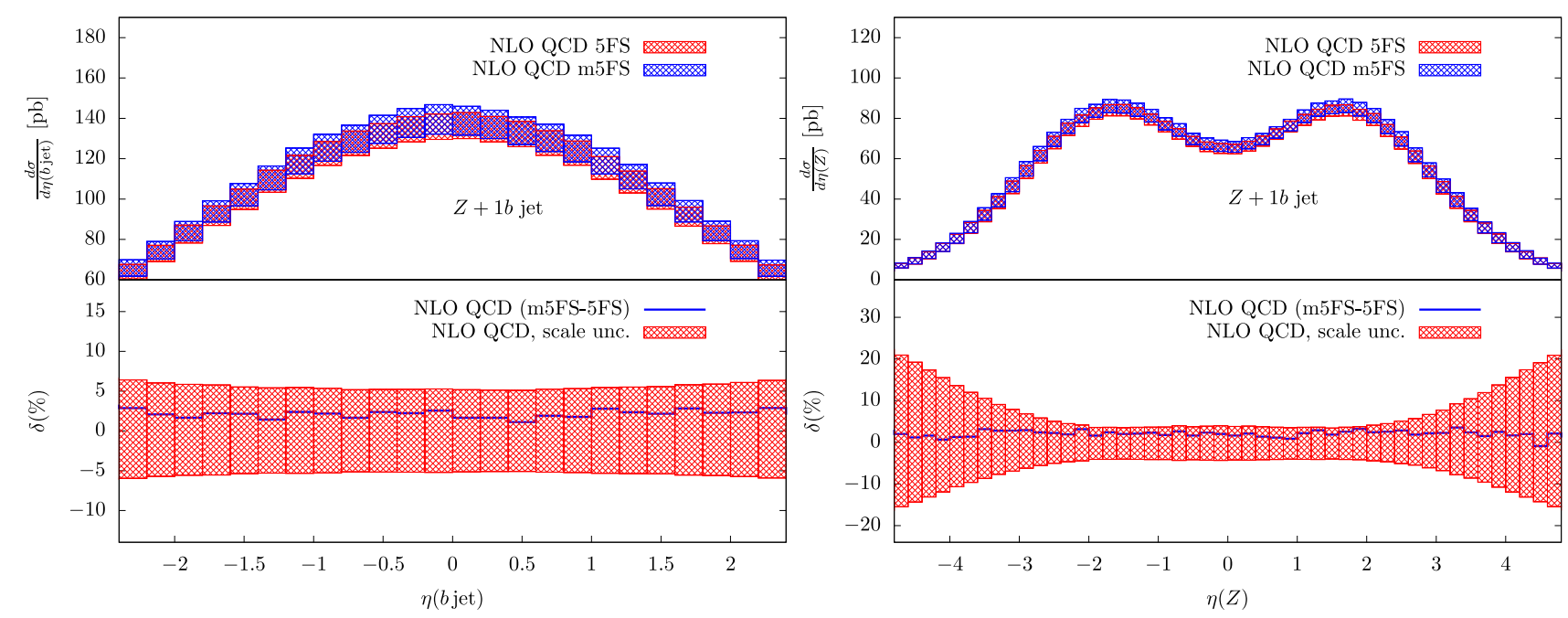

FIG. 7. The 5FS and m5FS NLO QCD predictions for differential distributions for the $Z$ and $b$-jet pseudorapidity. The lower plot shows the difference, $\delta_{\mathrm{mb}}$, together with the NLO QCD scale uncertainty. 
TABLE II. Total cross sections at LO and NLO (first row) including only QCD NLO corrections, only EW NLO corrections, or both (in the additive and multiplicative approaches), at the LHC with c.m. energy $13 \mathrm{TeV}$. The results obtained with $m_{b}=0$ at LO and NLO QCD are given in parentheses. The central values are obtained for $\mu_{r}=\mu_{f}=M_{Z}$ and the quoted uncertainties are calculated varying $\mu_{r}$ by a factor of 2 about the central value, for fixed $\mu_{f}=M_{Z}$. The relative impact of each NLO contribution, $\delta(\%)=\left(\sigma_{X} / \sigma_{\mathrm{LO}}-1\right) \times 100$ (for $X=$ NLO QCD, NLO EW, ...), is given in the second row. See text for more details. The errors reported in sub- and superscripts are purely from scale variation, while the statistical error (not reported) is on the last digit of the given results.

\begin{tabular}{lccccc}
\hline \hline & LO & NLO QCD & NLO EW & NLO QCD + EW & NLO QCD $\times$ EW \\
\hline$\sigma[\mathrm{pb}]$ & $389.73_{-37}^{+46}\left(392.66_{-37}^{+46}\right)$ & $537.7_{-29}^{+30}\left(526.9_{-28}^{+29}\right)$ & $383.40_{-36}^{+44}$ & $531.4_{-29}^{+30}$ & $529.2_{-29}^{+30}$ \\
$\delta[\%]$ & & $38(34)$ & -1.6 & 36 & 36 \\
\hline \hline
\end{tabular}

kind of NLO corrections and their combined effect on the LO cross section, in the second row of Table II we give the relative corrections as a percentage of the LO cross section, $\delta(\%)=\left(\sigma_{X} / \sigma_{\mathrm{LO}}-1\right) \times 100($ where $X=\mathrm{NLO} \mathrm{QCD}$, NLO EW, ...).

If the impact of NLO EW corrections on the total cross section is indicative of the average magnitude of their effect, a much more interesting result is their effect on distributions. Furthermore, it is important to compare the effect of NLO EW corrections to the residual theoretical uncertainty of NLO distributions, including both QCD and EW corrections, and estimate in particular whether NLO EW corrections are within the scale uncertainty of the corresponding NLO QCD corrections. With this in mind, in the following we will quantify the impact of EW corrections in terms of the following ratios:

$$
\delta_{\mathrm{EW}}^{\mathrm{add}}=\frac{\sigma_{\mathrm{NLO}}^{\mathrm{QCD}+\mathrm{EW}}-\sigma_{\mathrm{NLO}}^{\mathrm{QCD}}}{\sigma_{\mathrm{NLO}}^{\mathrm{QCD}}}=\frac{\sigma_{\mathrm{NLO}}^{\mathrm{QCD}+\mathrm{EW}}}{\sigma_{\mathrm{NLO}}^{\mathrm{QCD}}}-1
$$

and

$$
\delta_{\mathrm{EW}}^{\mathrm{pWod}}=\frac{\sigma_{\mathrm{NLO}}^{\mathrm{QCD} \times \mathrm{EW}}-\sigma_{\mathrm{NLO}}^{\mathrm{QCD}}}{\sigma_{\mathrm{NLO}}^{\mathrm{QCD}}}=\frac{\sigma_{\mathrm{NLO}}^{\mathrm{EW}}}{\sigma_{\mathrm{LO}}}-1,
$$

which correspond to the additive and multiplicative approaches of combining NLO QCD and EW cross sections, respectively.

Figures 3-5 illustrate the results of our calculation in terms of several distributions. In Fig. 3 we present the differential distributions for the transverse momentum of the final-state $b$-jet $\left[p_{T}(b\right.$ jet $\left.)\right]$ and $Z$ boson $\left[p_{T}(Z)\right]$ as well as their invariant mass $[\mathrm{M}(\mathrm{Z}, \mathrm{b}$ jet $)]$ in the region of low $p_{T}$ and low invariant mass, while the analogous distributions in the region of high $p_{T}$ and high invariant mass are given in Fig. 5. Given the large difference between the magnitude of the corresponding distributions at low and high momenta, we separate the two regions to illustrate the effects of EW corrections both at the peak (low- $p_{T} /$ mass region) and in the tail (high- $p_{T} /$ mass region) of such distributions. The upper plots of these figures show the comparison between the NLO distributions obtained including only QCD corrections or both QCD and EW corrections.
Each distribution is given as a band obtained by considering the renormalization-scale variation for fixed $\mu_{f}$, as explained earlier. For the sake of readability, in the upper plots we only compare to the additive combination of QCD and EW NLO corrections, while we consider both cases in the lower plots. Indeed, the lower plots of these figures illustrate both $\delta_{\mathrm{EW}}^{\text {add }}$ and $\delta_{\mathrm{EW}}^{\mathrm{prod}}$, as defined in Eqs. (22) and (23), and compare them to the bin-by-bin uncertainty of the NLO QCD cross section. The same information is provided in Fig. 4 for the $b$-jet and $Z$-boson pseudorapidity distributions $[\eta(\mathrm{b}$ jet $)$ and $\eta(Z)]$.

The results presented in Figs. 3-5 confirm that the effect of EW corrections on both $p_{T}$ and $\eta$ distributions are at the level of a few percent as for the total cross section (see Table II), apart from the high $p_{T}$ regions. The impact of the EW $O(\alpha)$ corrections on the LO cross sections [see Eq. (23)] can be seen in the relative corrections of the multiplicatively combined QCD and EW NLO corrections in the lower plots of Fig. 5, in particular at high $p_{T}$, where they reduce the central-scale LO cross section by $\sim 24 \%$ at $p_{T}=900 \mathrm{GeV}$. However, as discussed in Sec. II B these effects will have to be reexamined when experimental analyses require the inclusion of real $\mathrm{EW}$ gauge-boson radiation. In the low- $p_{T}$ region the impact of EW corrections is completely within the scale uncertainty of the differential NLO QCD cross section ${ }^{11}$ while at high $p_{T}$ EW corrections may be large enough to exceed the QCD uncertainty. For analyses focusing on the high- $p_{T}$ region this statement will have to be confirmed by dedicated studies of the theoretical uncertainty in realistic simulations that not only included the effect of scale variation. In the case of pseudorapidity distributions, both the additive and multiplicative combinations of QCD and EW NLO corrections show that the effect of EW corrections is still hidden in the NLO QCD uncertainty affecting these distributions, with the central $\eta(Z)$ region being the least affected.

\footnotetext{
${ }^{11}$ We notice that while the $p_{T}^{b}$ spectrum is cut at low $p_{T}$ by a tagging cut, the $p_{T}^{Z}$ spectrum at NLO can extend to vanishing $p_{T}$. The unusually large scale dependence in the region around the $b$-jet $p_{T}$ cut is due to well known instabilities in matching the LO and NLO phase spaces.
} 
Following the same logic, we quantify the effect of switching from a massless 5FS to a m5FS in terms of the ratio

$$
\delta_{\mathrm{mb}}=\frac{\sigma_{\mathrm{NLO}}^{\mathrm{QCD}, \mathrm{m} 5 \mathrm{FS}}-\sigma_{\mathrm{NLO}}^{\mathrm{QCD}, 5 \mathrm{FS}}}{\sigma_{\mathrm{NLO}}^{\mathrm{QCD}, 5 \mathrm{FS}}}=\frac{\sigma_{\mathrm{NLO}}^{\mathrm{QCD}, \mathrm{m} 5 \mathrm{FS}}}{\sigma_{\mathrm{NLO}}^{\mathrm{QCD}, 5 \mathrm{FS}}}-1,
$$

representing the fractional change in the NLO QCD cross sections in going from the 5FS to the m5FS. This ratio is given in the lower plots of Figs. 6 and 7, where we present results for the final-state $b$-jet and $Z$-boson $p_{T}$ distributions, and their invariant-mass and pseudorapidity distributions. In these figures, the upper plots give the explicit form of the distributions in the 5FS and the m5FS, together with their bin-by-bin scale uncertainty. Most of the observed mass effects still lie within the uncertainty of the differential NLO QCD cross sections, in particular for $p_{T}$ and invariant-mass distributions. The largest deviations are at the level of a few percent and are concentrated as expected in the low- $p_{T} /$ mass region. On the other hand, the pseudorapidity distributions are sensitive to mass effects over their entire range. We do indeed expect that a modification of the initial-state kinematics can lead to modifications of the final-state angular distributions. This corroborates our original motivation for a consistent implementation of a m5FS in Monte Carlo event generators.

Finally, it is clear from our study that better control of higher-order QCD corrections is still a limiting factor in achieving further theoretical accuracy in the prediction of $Z+b$-jet production. The residual still sizable scale dependence of both total and differential cross sections in the m5FS (and the 5FS) does not come as a surprise since in the calculation of the $O\left(\alpha_{s}^{2} \alpha\right)$ part of the NLO cross section for $b g \rightarrow Z b$ new important channels (such as $g g \rightarrow Z b \bar{b}$ ) appear for the first time, and introduce a large Born-like scale dependence in the NLO QCD cross section. As already emphasized in Sec. I, this large scale dependence could be greatly reduced by calculating the NNLO QCD corrections to $b g \rightarrow Z b$, where the known NLO QCD corrections to $g g \rightarrow Z b \bar{b}$ [25] contribute, possibly retaining the $b$ quark initial-state mass dependence.

\section{CONCLUSIONS}

In this paper we have considered the production of a $Z$ boson with at least one $b$ jet as a phenomenologically interesting testing ground to assess the impact of finite initial-state $b$-quark masses and to evaluate the relevance of a still missing piece of NLO corrections, the combined QCD and EW NLO corrections. We have consistently implemented the initial-state massive kinematics and the corresponding matching with existing PDF, setting the stage for a consistent development of a massive 5FS, which should provide the correct interface between fixed-order NLO calculations and parton-shower Monte Carlo event generators. We have presented the first calculation of the first-order EW corrections to $b g \rightarrow Z b$, using the NLOX one-loop provider, and implementing real photon-emission corrections using both a phase-space slicing method and dipole subtraction with massive initial-state dipoles. EW and QCD NLO corrections have been combined using both additive and multiplicative approaches.

Both mass effects and NLO EW corrections are small effects, compared to the size of NLO QCD corrections, and are mostly within the uncertainty of the NLO QCD cross section. Still, there are clear indications of their impact, in particular on the entire spectrum of angular distributions, and in complementary transverse-momentum regions: EW corrections mainly affect the high- $p_{T}$ region of both $b$-jet and $Z$ boson $p_{T}$, while $b$-quark mass effects are more pronounced in the low- $p_{T}$ regions.

Given the phenomenological relevance of $Z+b$-jet production, both as background to Higgs-boson precision measurements and new physics searches, as well as a potential candidate for a direct precision measurement of the $b$-quark PDF, we should aim to reduce the theoretical uncertainty in the future. In this respect, it is clear that the inclusion of NNLO QCD corrections will greatly help to mitigate unphysical scale dependencies. At the same time, the consistent inclusion of $b$-quark mass effects in existing PDF sets, where the $b$-quark mass only plays the role of an IR regulator so far, can only improve our control of the Monte Carlo generation of $b$ initiated processes.

\section{ACKNOWLEDGMENTS}

We thank Pavel Nadolsky, Fred Olness, and Dave Soper for discussions. L. R. and D. F. particularly thank Fernando Febres-Cordero for his interest in clarifying some technical aspects of the implementation of massive initial-state dipoles. The work of S. H., S. Q., L. R., and C. R. has been and is supported in part by the U.S. Department of Energy under Grant No. DESC0010102. The work of D. W. is supported in part by the U.S. National Science Foundation under Grant No. NSF-PHY-1417317. S. H., L. R., C. R., and D. W. are grateful for the hospitality of the Kavli Institute for Theoretical Physics (KITP) during the workshop on LHC Run II and the Precision Frontier at which part of this work was prepared. Their research at the KITP was supported in part by the National Science Foundation under Grant No. NSF PHY-1748958. L. R. also thanks the Aspen Center for Physics for the hospitality offered while parts of this work were being completed. 
[1] T. Aaltonen et al. (CDF Collaboration), Phys. Rev. D 79, 052008 (2009).

[2] V. M. Abazov et al. (D0 Collaboration), Phys. Rev. D 87, 092010 (2013).

[3] G. Aad et al. (ATLAS Collaboration), Phys. Lett. B 706, 295 (2012).

[4] G. Aad et al. (ATLAS Collaboration), J. High Energy Phys. 10 (2014) 141.

[5] S. Chatrchyan et al. (CMS Collaboration), J. High Energy Phys. 06 (2012) 126.

[6] S. Chatrchyan et al. (CMS Collaboration), J. High Energy Phys. 12 (2013) 039.

[7] S. Chatrchyan et al. (CMS Collaboration), J. High Energy Phys. 06 (2014) 120.

[8] V. Khachatryan et al. (CMS Collaboration), Eur. Phys. J. C 77, 751 (2017).

[9] R. Aaij et al. (LHCb Collaboration), J. High Energy Phys. 01 (2015) 064.

[10] J. M. Campbell, R. K. Ellis, F. Febres Cordero, F. Maltoni, L. Reina, D. Wackeroth, and S. Willenbrock, Phys. Rev. D 79, 034023 (2009).

[11] J. Campbell, F. Caola, F. Febres Cordero, L. Reina, and D. Wackeroth, Phys. Rev. D 86, 034021 (2012).

[12] F. Maltoni, G. Ridolfi, and M. Ubiali, J. High Energy Phys. 07 (2012) 022.

[13] S. Forte, D. Napoletano, and M. Ubiali, Phys. Lett. B 751, 331 (2015).

[14] M. Lim, F. Maltoni, G. Ridolfi, and M. Ubiali, J. High Energy Phys. 09 (2016) 132.

[15] S. Forte, D. Napoletano, and M. Ubiali, Phys. Lett. B 763, 190 (2016).

[16] M. Bonvini, A. S. Papanastasiou, and F. J. Tackmann, J. High Energy Phys. 11 (2015) 196.

[17] M. Bonvini, A. S. Papanastasiou, and F. J. Tackmann, J. High Energy Phys. 10 (2016) 053.

[18] F. Krauss, D. Napoletano, and S. Schumann, Phys. Rev. D 95, 036012 (2017).

[19] F. Febres Cordero and L. Reina, Int. J. Mod. Phys. A 30, 1530042 (2015).

[20] J. M. Campbell, R. K. Ellis, F. Maltoni, and S. Willenbrock, Phys. Rev. D 69, 074021 (2004).

[21] J. M. Campbell, R. K. Ellis, and C. Williams, MCFM, from v.8.0 (2015), http://mcfm.fnal.gov.

[22] A. Gehrmann-De Ridder, T. Gehrmann, E. W. N. Glover, A. Huss, and T. A. Morgan, Phys. Rev. Lett. 117, 022001 (2016).

[23] R. Boughezal, J. M. Campbell, R. K. Ellis, C. Focke, W. T. Giele, X. Liu, and F. Petriello, Phys. Rev. Lett. 116, 152001 (2016).

[24] F. Febres Cordero, L. Reina, and D. Wackeroth, Phys. Rev. D 78, 074014 (2008).

[25] F. Febres Cordero, L. Reina, and D. Wackeroth, Phys. Rev. D 80, 034015 (2009).

[26] J. H. Kuhn, A. Kulesza, S. Pozzorini, and M. Schulze, Nucl. Phys. B727, 368 (2005).

[27] A. Denner, S. Dittmaier, T. Kasprzik, and A. Mück, Proc. Sci., ICHEP2010 (2010) 058.

[28] A. Denner, S. Dittmaier, T. Kasprzik, and A. Mück, J. High Energy Phys. 06 (2011) 069.
[29] W. Hollik, B. A. Kniehl, E. S. Scherbakova, and O. L. Veretin, Nucl. Phys. B900, 576 (2015).

[30] S. Kallweit, J. M. Lindert, P. Maierhofer, S. Pozzorini, and M. Schönherr, J. High Energy Phys. 04 (2016) 021.

[31] J. C. Collins, Phys. Rev. D 58, 094002 (1998).

[32] M. Aivazis, J. C. Collins, F. I. Olness, and W.-K. Tung, Phys. Rev. D 50, 3102 (1994).

[33] M. Krämer, F. I. Olness, and D. E. Soper, Phys. Rev. D 62, 096007 (2000).

[34] F. Krauss and D. Napoletano, arXiv:1712.06832.

[35] S. Honeywell, S. Quackenbush, L. Reina, and C. Reuschle, NLOX release note and manual (to be published).

[36] S. Dittmaier, Nucl. Phys. B565, 69 (2000).

[37] Z. Nagy and D. E. Soper, J. High Energy Phys. 06 (2014) 179.

[38] S. Actis, A. Denner, L. Hofer, J.-N. Lang, A. Scharf, and S. Uccirati, Comput. Phys. Commun. 214, 140 (2017).

[39] T. Hahn, Comput. Phys. Commun. 168, 78 (2005).

[40] L. Reina and T. Schutzmeier, J. High Energy Phys. 09 (2012) 119.

[41] L. Reina and T. Schutzmeier, Proc. Sci., LL2012 (2012) 021.

[42] J. Bendavid et al., Les Houches 2017: Physics at TeV Colliders Standard Model Working Group Report [arXiv:1803.07977].

[43] P. Nogueira, J. Comput. Phys. 105, 279 (1993).

[44] J. Kuipers, T. Ueda, J. A. M. Vermaseren, and J. Vollinga, Comput. Phys. Commun. 184, 1453 (2013).

[45] G. Passarino and M. J. G. Veltman, Nucl. Phys. B160, 151 (1979).

[46] A. Denner and S. Dittmaier, Nucl. Phys. B734, 62 (2006).

[47] A. van Hameren, Comput. Phys. Commun. 182, 2427 (2011).

[48] R. K. Ellis and G. Zanderighi, J. High Energy Phys. 02 (2008) 002.

[49] S. Carrazza, R. K. Ellis, and G. Zanderighi, Comput. Phys. Commun. 209, 134 (2016).

[50] A. Denner, Fortschr. Phys. 41, 307 (1993).

[51] A. Denner, S. Dittmaier, M. Roth, and L. H. Wieders, Nucl. Phys. B724, 247 (2005); B854, 504(E) (2012).

[52] W. F. L. Hollik, Fortschr. Phys. 38, 165 (1990).

[53] S. Dittmaier and M. Krämer, Phys. Rev. D 65, 073007 (2002).

[54] J. Butterworth et al., Les Houches 2013: Physics at TeV Colliders Standard Model Working Group Report [arXiv:1405.1067].

[55] J. C. Collins, F. Wilczek, and A. Zee, Phys. Rev. D 18, 242 (1978).

[56] P. Nason, S. Dawson, and R. K. Ellis, Nucl. Phys. B303, 607 (1988).

[57] B. W. Harris and J. F. Owens, Phys. Rev. D 65, 094032 (2002).

[58] U. Baur, Phys. Rev. D 75, 013005 (2007).

[59] M. Ciafaloni, P. Ciafaloni, and D. Comelli, Phys. Rev. Lett. 84, 4810 (2000).

[60] M. Ciafaloni, P. Ciafaloni, and D. Comelli, Nucl. Phys. B589, 359 (2000).

[61] A. Denner, S. Dittmaier, T. Kasprzik, and A. Mück, J. High Energy Phys. 08 (2009) 075. 
[62] P. Kotko and W. Slominski, Phys. Rev. D 86, 094008 (2012).

[63] J. Amundson, C. Schmidt, W.-K. Tung, and X. Wang, J. High Energy Phys. 10 (2000) 031.

[64] W.-K. Tung, S. Kretzer, and C. Schmidt, J. Phys. G 28, 983 (2002).

[65] S. Kretzer and I. Schienbein, Phys. Rev. D 58, 094035 (1998).

[66] T. Binoth et al. (SM and NLO Multileg Working Group Collaboration), Les Houches 2009: Physics at TeV Colliders Standard Model Working Group Report [arXiv: 1003.1241].

[67] R. D. Ball, V. Bertone, M. Bonvini, S. Forte, P. Groth Merrild, J. Rojo, and L. Rottoli, Phys. Lett. B 754, 49 (2016).

[68] R. D. Ball, M. Bonvini, and L. Rottoli, J. High Energy Phys. 11 (2015) 122.

[69] R. D. Ball, V. Bertone, M. Bonvini, S. Carrazza, S. Forte, A. Guffanti, N. P. Hartland, J. Rojo, and
L. Rottoli (NNPDF Collaboration), Eur. Phys. J. C 76, 647 (2016).

[70] C. Schmidt, J. Pumplin, D. Stump, and C. P. Yuan, Phys. Rev. D 93, 114015 (2016).

[71] S. Dulat, T.-J. Hou, J. Gao, M. Guzzi, J. Huston, P. Nadolsky, J. Pumplin, C. Schmidt, D. Stump, and C. P. Yuan, Phys. Rev. D 93, 033006 (2016).

[72] U. Baur, S. Keller, and D. Wackeroth, Phys. Rev. D 59, 013002 (1998).

[73] K. P. O. Diener, S. Dittmaier, and W. Hollik, Phys. Rev. D 72, 093002 (2005).

[74] B. A. Kniehl, G. Kramer, I. Schienbein, and H. Spiesberger, Eur. Phys. J. C 41, 199 (2005).

[75] K. A. Olive et al. (Particle Data Group Collaboration), Chin. Phys. C 38, 090001 (2014).

[76] A. Sirlin, Phys. Rev. D 22, 971 (1980).

[77] J. M. Campbell, D. Wackeroth, and J. Zhou, Phys. Rev. D 94, 093009 (2016). 